high CRP and DAS28, swollen joint count, LDL cholesterol level, menopause, thickness of CIMT and tibial artery. Were identify a significant correlation between tibial artery thickness and age, BMI, RA duration, systolic and diastolic blood pressure, cholesterol, menopause $(p<0.01, \times 2=26.18)$. Also, we identified a significant relationship between tibial artery thickness and changes of the intima morphology $(x 2=31,64 ; p<0,01)$, carotid plaques $(x 2=26,179 ; p<0,01)$, sclerosis of the heart valves $(x 2=25,78 ; p<0,01)$. A less significant relationship was between $\operatorname{cIMT}(x 2=8,507 ; p<0,01)$. The DAS28, age $(p<0.001)$, thickness of cIMT and tibial artery $(p<0.001)$ are predictive factors for the development of carotid plaques. The predictive factors for tibial artery thickness in female patients $<45$ age are: high CRP $(p<0.004)$ and DAS28 $(p<0.03)$, anti-CCP positivity $(p<0.04)$, Vps internal carotid artery $(p<0.04)$

Conclusions: The results of our study indicate that high CRP and DAS28 score, swollen joint count, LDL cholesterol level, menopause, thickness of cIMT and tibial artery associated with the increased cardiovascular risk. Tibial artery thickness and Vps internal carotid artery may be assessed like the new predictive factors of $\mathrm{CV}$ diseases in RA female patient

Acknowledgements: None

Disclosure of Interest: None declared

DOI: 10.1136/annrheumdis-2017-eular.6388

\section{FRI0161 NON-INVASIVE ASSESSMENT OF MYOCARDIAL PERFUSION IN PATIENTS WITH RHEUMATOID ARTHRITIS}

P. Anyfanti ${ }^{1}$, N. Koletsos ${ }^{1}$, A. Triantafyllou ${ }^{1}$, S. Chatzimichailidou ${ }^{2}$, G. Triantafyllou ${ }^{1}$, P. Panagopoulos ${ }^{1}$, E. Gkaliagkousi ${ }^{1}$, S. Aslanidis ${ }^{2}$, S. Douma ${ }^{1} .{ }^{1}$ rd Department of Internal Medicine, Papageorgiou Hospital; ${ }^{2} 2 n d$ Propedeutic Department of Internal Medicine, Hippokration Hospital, Aristotle University of Thessaloniki, Thessaloniki, Greece

Background: Cardiac involvement among patients with rheumatoid arthritis (RA) is common, potentially life-threatening, but often underdiagnosed at presymptomatic stages. Subendocardial viability ratio (SEVR) reflects microvascular coronary perfusion, as it correlates with the ratio of subepicardial to subendocardial blood flow, that can be non-invasively estimated by applanation tonometry. Although it has been studied as a surrogate measure of myocardial perfusion in high-cardiovascular risk populations, it remains unclear whether it is affected in RA patients.

Objectives: The purpose of the study was to compare SEVR between RA patients and healthy controls. We additionally sought predictors of SEVR in RA among a wide range of disease-related parameters, hemodynamic factors, and markers of atherosclerosis, arteriosclerosis, and endothelial dysfunction.

Methods: Consecutive patients with RA and healthy, nontreated volunteers were recruited. SEVR was estimated from applanation tonometry with the Sphygmocor device (AtCor Medical, Sydney, Australia), which was also used to evaluate arterial stiffness (aortic index, Alx; pulse wave velocity, PWV). In the RA group, carotid atherosclerosis was additionally evaluated by ultrasound (carotid intima-media thickness, cIMT); cardiac and hemodynamic parameters by impedance cardiography, and endothelial dysfunction by measurement of asymmetric dimethylarginine (ADMA) in serum samples.

Results: A total of 122 participants, 91 RA patients and 31 controls, were studied. SEVR was significantly lower among RA patients compared to controls (141.4 \pm 21.9 vs $153.1 \pm 18.7 \%, p=0.005)$, and the same was observed when the subgroup of RA patients without cardiovascular comorbidities $(n=29)$ was studied separately $(139.7 \pm 21.7$ vs $153.1 \pm 18.7 \%, p=0.013)$. In the univariate analysis, SEVR significantly correlated with cardiac and hemodynamic parameters, but not with PWV, Alx, CIMT, ADMA, or disease-related parameters. In the linear regression analysis accounting for sex, statin use, markers of atherosclerosis, cardiac, and hemodynamic parameters, female gender $(p=0.007)$, blood pressure $(p=0.028)$, heart rate $(p=0.025)$, cholesterol levels $(p=0.008)$, cardiac index $(p<0.001)$, and left ventricular ejection time $(p=0.004)$ were identified as independent predictors of SEVR among patients with RA.

Conclusions: Patients with RA exhibit lower values of SEVR compared to healthy individuals, suggesting a disturbed balance between oxygen supply and demand that might provide an additional pathophysiological link for the increased cardiovascular burden in RA. Cardiac and hemodynamic parameters, rather than markers of atherosclerosis, arteriosclerosis, and endothelial dysfunction, may be useful as predictors of impaired myocardial perfusion in RA.

References:

[1] Buckberg GD, et al. Experimental subendocardial ischemia in dogs with normal coronary arteries. Circ Res 1972;30:67-81.

[2] Sarnoff SJ, et al. Hemodynamic determinants of oxygen consumption of the heart with special reference to the tension-time index. Am J Physiol 1958;192:148-56.
[3] Tsiachris D, et al. Subendocardial viability ratio as an index of impaired coronary flow reserve in hypertensives without significant coronary artery stenoses. J Hum Hypertens 2012;26:64-70.

Disclosure of Interest: None declared

DOI: 10.1136/annrheumdis-2017-eular.4903

\section{FRI0162 ANTIBODY RESPONSE TO 13-VALENT PNEUMOCOCCAL CONJUGATE VACCINE IS NOT IMPAIRED IN PATIENTS WITH RHEUMATOID ARTHRITIS (RA) OR SJOGRENS SYNDROME WITHOUT DMARD TREATMENT COMPARED TO CONTROLS}

P. Nived $^{1,2}$, J. Nagel ${ }^{1}$, T. Saxne ${ }^{1}$, P. Geborek ${ }^{1}$, T. Mandl ${ }^{3}$, L. Skattum ${ }^{4,5}$, M.C. Kapetanovic ${ }^{1} .{ }^{1}$ Department of Clinical Sciences Lund, Section of Rheumatology, Skåne University Hospital, Lund University, Lund; ${ }^{2}$ Clinic for Infectious Diseases, KRYH, Kristianstad; ${ }^{3}$ Department of Clinical Sciences Malmö, Section of Rheumatology, Skåne University Hospital, Lund University, Malmö; ${ }^{4}$ Clinical Immunology and Transfusion Medicine, Region Skåne;

${ }^{5}$ Department of Laboratory Medicine, Section of Microbiology, Immunology and Glycobiology, Lund University, Lund, Sweden

Objectives: To investigate if antibody response and functionality of antibodies following immunization with 13-valent pneumococcal conjugate vaccine (PCV13) is impaired in patients with rheumatoid arthritis (RA) and primary Sjogrens syndrome (pSS) without treatment, with methotrexate (MTX) or hydroxychloroquine (HCQ), compared to healthy controls.

Methods: In total, 61 patients with RA (51 without DMARD and 10 on MTX), 23 patients with pSS (18 without DMARD and 5 on $\mathrm{HCQ}$ ) and 49 controls were vaccinated with a single dose $(0.5 \mathrm{ml})$ PCV13 intramuscularly. Pre- and postvaccination serotype-specific antibody concentrations for pneumococcal serotypes $6 \mathrm{~B}$ and $23 \mathrm{~F}$ and functionality of antibodies $(23 \mathrm{~F}$ ) were determined in serum samples taken immediately before and 4-6 weeks after vaccination using ELISA and opsonophagocytic activity (OPA) assay, respectively. Proportions of individuals with positive antibody response (i.e. $\geq 2$-fold increase from prevaccination concentrations) for both serotypes were calculated and groups were compared using Chi2 test. Percentage change (pre- to postvaccination) in OPA was calculated and groups were compared using Mann-Whitney $\mathrm{U}$ test.

Results: Pre- to postvaccination antibody concentrations (Table) increased significantly for both serotypes and in both patient and control groups $(p<0.001)$. Antibody response to serotypes $6 \mathrm{~B}$ and $23 \mathrm{~F}$ was decreased in patients with $\mathrm{RA}$ on MTX treatment (both $p<0.01$ ), but not in RA without DMARD or pSS with or without $\mathrm{HCQ}$, compared to controls (Figure). Proportions of antibody responders to both serotypes did not differ significantly between groups RA without DMARD $(51 \%)$, pSS $(48 \%)$ and controls (55\%). After vaccination, OPA increased in groups RA without DMARD $(p<0.001)$, pSS without DMARD $(p=0.01)$ and controls $(p<0.001)$, but did not change significantly in patients with RA on MTX and pSS on HCQ. The mean percentage change in OPA was lower in RA on MTX $(1.8 \%$, $p=0.01)$, RA without DMARD $(8.9 \%, p<0.01)$ and $\mathrm{pSS}$ on $\mathrm{HCQ}(-1.5 \%, p=0.01)$, but not in pSS without DMARD (11.0\%), compared to controls (17.9\%).

Figure. Antibody response to vaccination.

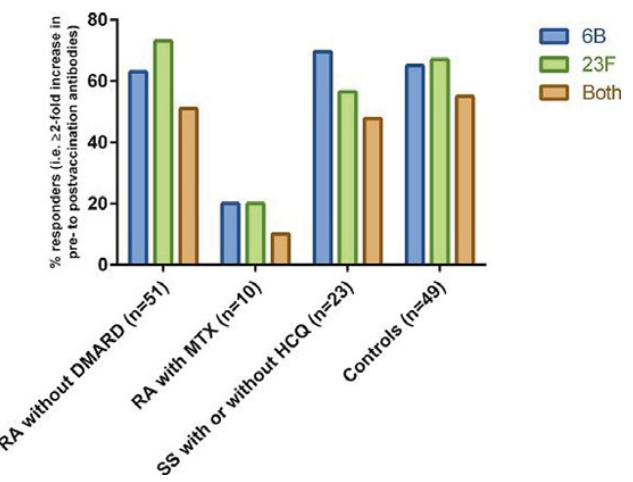

Conclusions: Pneumococcal conjugate vaccine is immunogenic in patients with $\mathrm{RA}$ and $\mathrm{pSS}$ without DMARD treatment. Both antibody response and functionality of antibodies is impaired in patients with RA on MTX.

Disclosure of Interest: None declared

DOI: 10.1136/annrheumdis-2017-eular.2146

Abstract FRI0162 - Table 1. Group characteristics and serotype-specific antibody geometric mean levels (GMLs, $\mu \mathrm{g} / \mathrm{mL}$ )

\begin{tabular}{|c|c|c|c|c|c|}
\hline & RA without DMARD $(n=51)$ & RA on MTX $(n=10)$ & Sjogrens syndrome without DMARD $(n=18)$ & Sjogrens syndrome on $\mathrm{HCQ}(\mathrm{n}=5)$ & Controls $(n=49)$ \\
\hline Age (mean; range) years & $65.2(35-87)$ & $65.3(39-79)$ & $58.9(26-90)$ & $61.0(33-80)$ & $50.6(17-85)$ \\
\hline Sex (\% female) & 78.4 & 70.0 & 88.9 & 100.0 & 63.3 \\
\hline GML $6 \mathrm{~B}$ prevaccination $(95 \% \mathrm{Cl})$ & $0.6(0.4-1.0)$ & $1.3(0.5-2.9)$ & $0.6(0.2-1.5)$ & $0.6(0.2-1.7)$ & $0.8(0.5-1.3)$ \\
\hline GML $6 \mathrm{~B}$ postvaccination $(95 \% \mathrm{Cl})$ & $3.3(1.8-6.0)$ & $2.1(0.8-5.6)$ & $2.4(1.1-5.4)$ & $1.7(0.2-11.4)$ & $3.1(1.9-5.0)$ \\
\hline GML $23 \mathrm{~F}$ prevaccination $(95 \% \mathrm{Cl})$ & $0.5(0.3-0.8)$ & $1.0(0.3-3.5)$ & $0.8(0.4-1.6)$ & $0.4(0.2-1.2)$ & $0.6(0.4-0.9)$ \\
\hline GML $23 \mathrm{~F}$ postvaccination $(95 \% \mathrm{Cl})$ & $2.4(1.5-3.9)$ & $1.7(0.7-3.9)$ & $3.6(1.4-9.1)$ & $1.1(0.2-6.6)$ & $3.3(2.0-5.5)$ \\
\hline
\end{tabular}

